\title{
O ESTUDO DA MORBIMORTALIDADE NO BRASIL SOB O ENFOQUE DAS RELAÇÕES ‘POPULAÇÃO E AMBIENTE’*
}

Alex Manetta ${ }^{* *}$

Resumo: A análise das especificidades do padrão da morbimortalidade, no Brasil, traz considerações importantes sobre suas componentes demográficas e sugere impactos crescentes das mudanças ambientais na saúde humana, na medida em que o processo de transição urbano-industrial avança. Na busca por resultados mais precisos, entretanto, conclui-se sobre a relevância de algumas das condições básicas para a continuidade dos estudos sobre população e ambiente: 1) a necessidade de avançar na geração e no tratamento espacial de dados e 2) a tendência em avaliar impactos locais das mudanças ambientais, evitando generalizações excessivas.

Palavras-chave: Morbimortalidade. Dinâmica demográfica. Ambiente. Urbanização. Brasil.

The study of morbidity and mortality in Brazil under the approach of relations 'population and environment'

Abstract: The analysis of the morbimortality patterns, in Brazil, has important considerations on their demographic components and suggests increasing impacts of environmental change on human health, to the extent that the process of urbanindustrial transition progresses. In the search for more accurate results, however, it is concluded on the relevance of some of the basic conditions for the continuation of studies about population and environment: 1) the need to advance on the generation and spatial data processing and 2) the tendency to assess local impacts of environmental change, avoiding excessive generalizations.

Keywords: Morbimortality. Demographic dynamics. Environment. Urbanization. Brazil.

* Agradecimento especial a Roberto L. do Carmo, a Igor C. Johansen e aos pareceristas da Revista Idéias, pelas preciosas colaborações na discussão do presente tema.

** Instituto de Filosofia e Ciências Humanas, Universidade Estadual de Campinas - Unicamp, Campinas-SP, Brasil (alexmanetta@hotmail.com).

Recebido em: 27/03/2015 - Aceito em: 23/07/2015. 
$|40|$

O estudo da morbimortalidade no Brasil sob o enfoque...

El estudio de la morbimortalidad en Brasil bajo el enfoque de las relaciones 'Población y Medio Ambiente'

Resumen: El análisis de las especificidades de los patrones de morbimortalidad, en Brasil, trae consideraciones importantes acerca de sus componentes demográficos y sugiere crecientes impactos de los cambios ambientales sobre la salud humana, en la medida en que el proceso de transición urbano-industrial avanza. En la búsqueda de resultados más exactos, sin embargo, se concluye acerca de la relevancia de algunas de las condiciones básicas para la continuidad de los estudios sobre población y medio ambiente: 1) la necesidad de avanzar en la generación y tratamiento espacial de los datos y 2) la tendencia a evaluar los impactos locales de los cambios ambientales, evitando las generalizaciones excesivas.

Palabras clave: Morbimortalidad. Dinámica demográfica. Medio ambiente. Urbanización. Brasil.

\section{Introdução}

Durante os anos 1970, quando aflorou a questão ambiental, a demografia não possuía uma contribuição bem formulada ao debate, que fosse além da perspectiva neomalthusiana ${ }^{1}$. Por isso, em décadas recentes, o campo de estudos sobre população

1 Os neomalthusianos assimilaram os receios de Malthus e propuseram soluções para a questão da escassez de recursos a partir da redução do ritmo de crescimento populacional. Malthus sustentou que a população tenderia a crescer em progressão geométrica, enquanto a produção de alimentos tenderia a crescer em progressão aritmética, em uma dinâmica que levaria, sem dúvidas, à escassez (MADEIRA, 2008). Não é difícil, entretanto, reconhecer que essa teoria apresenta graves problemas, já que admite um crescimento linear da produção agrícola, ignorando as possibilidades dadas pela evolução técnica, ao mesmo tempo em que considera o aumento exponencial da população, em um momento no qual a dinâmica demográfica passou a apresentar claros sinais de mudanças, com quedas nas taxas de mortalidade, de fecundidade e de crescimento da população total (VANWEY; OSTROM; MERETSKY, 2009). Os neomalthusianos, apesar de vivenciarem outro momento histórico, sustentam uma versão simplista da relação populaçãoambiente, com foco na pressão dos números sobre recursos, privilegiando leituras enviesadas da realidade, através de conceitos amplamente superados, como o da 'explosão demográfica', por exemplo, reduzindo a complexidade deste debate (HOGAN, 2007). 
e ambiente (P-A) tem sido chamado a superar, mesmo que de forma parcial, o enfoque predominante. Os caminhos encontrados partem dos estudos centrados no volume e no ritmo de crescimento da população, sem desconsiderar sua atual relevância, em direção à abordagem de cada uma das componentes da dinâmica demográfica, em suas relações com as alterações ambientais (HOGAN, 2000a).

A demografia corresponde ao estudo científico da população. Inclui aspectos como tamanho, ritmo de crescimento, distribuição espacial e composição por sexo e idade, além dos fatores que influenciam na alteração de suas tendências. Entretanto, as componentes da dinâmica demográfica, estritamente definidas, são: a fecundidade, a mortalidade e a migração (SWANSON; SIEGEL, 1976).

No Brasil, embora se reconheça que a queda nas taxas de mortalidade mantenha associações explícitas com a dinâmica ambiental, uma vez que se dá a partir de melhorias nas condições de saneamento urbano, de nutrição e de acesso a outras inovações técnicas, científicas e socioeconômicas, o estudo das relações entre ambiente e mortalidade tem recebido pouca atenção por parte dos demógrafos (HOGAN, 2007).

No que diz respeito aos estudos sobre P-A, as atenções se concentraram nas relações 'ambiente $\rightarrow$ saúde' (LUTZ; PRSKAWETZ; SANDERSON, 2002), salientando eventos de alteração ${ }^{2}$ ambiental como causas de agravos específicos à saúde humana.

A mensuração e a análise comparativa de dados, visando a identificação de relações estatísticas entre padrões de

\footnotetext{
${ }^{2}$ Faz-se referência: 1) a processos vigentes em amplas escalas espaciais, como a urbanização; a industrialização e as alterações climáticas globais; e 2) a eventos vigentes em escalas relativamente restritas, como no caso da contaminação ambiental por deposição final inadequada de rejeitos químicos ou domiciliares; de desastres após eventos climáticos intensos; e de práticas que propiciam a proliferação de vetores biológicos contaminados e a introdução de novos sorotipos de doenças na população humana; por exemplo.
} 
$\mid 42$ |

O estudo da morbimortalidade no Brasil sob o enfoque...

morbimortalidade e as condições ambientais, no entanto, têm sido bastante comprometidas, pela inexistência de registros capazes de serem utilizados de forma precisa em modelos e sistemas de informação geográfica (HOGAN, 2000b).

No estudo da incidência de doenças endêmicas/epidêmicas cujos vetores são bem conhecidos, como a malária e a dengue, por exemplo, informações sobre o regime climático, o uso do solo, a qualidade e a cobertura dos serviços e infraestruturas urbanas, assim como sobre a ocupação e a mobilidade espacial da população, trazem componentes relevantes para o reconhecimento de relações entre processos de alteração ambiental e agravos à saúde humana, embora sua comprovação empírica seja ainda difícil.

No estudo da morbimortalidade por neoplasias, cardiopatias e doenças respiratórias, por seu caráter multicausal, reconhece-se uma complexidade extra, tanto no registro mais preciso da principal causa de morte quanto no estabelecimento de relações causais diretas com a exposição a substâncias químicas, alterações comportamentais ou vetores biológicos nocivos à saúde, tornando difícil a distinção entre o peso relativo dos diversos fatores que levam ao aumento da incidência dessas doenças.

Reconhecendo as dificuldades na geração de dados demográficos e ambientais para um tratamento estatístico refinado, assim como as limitações das bases de dados disponíveis, salientase o potencial do padrão de morbimortalidade como indicador útil no campo de estudos sobre P-A no Brasil.

\section{A análise da morbimortalidade no campo de estudos sobre P-A}

Os dados de morbimortalidade continuam sendo um caminho para o estudo das relações entre ambiente e saúde, de modo que elevados níveis de incidência doenças diarreicas, por exemplo, indicam más condições de saneamento básico domiciliar para parcelas significativas das famílias. Já a elevação da incidência de cânceres e de doenças cardíacas e respiratórias apresenta relações com a exposição a uma ampla gama de contaminantes 
químicos, presentes no ar, na água e nos solos, nos alimentos e em outros produtos largamente consumidos no contexto da transição urbano-industrial brasileira (HOGAN, 2000b).

A abordagem sobre P-A, no que diz respeito ao estudo das principais causas de morbimortalidade, por sexo e idade, salienta também que os recém-nascidos e as crianças pequenas, juntamente com os idosos, tendem a sofrer de forma mais aguda os efeitos da poluição atmosférica e das doenças diarreicas, agravadas pela falta de saneamento básico. Já os adolescentes e jovens adultos, do sexo masculino, tendem a responder ao estresse urbano com mais violência que as mulheres (HOGAN, 2007).

Desse ponto de vista, o estudo das relações entre o padrão de morbimortalidade e as condições ambientais demanda a observação de processos e de eventos com relevante potencial de agravo ou de melhoria nas condições de saúde da população, cujos impactos sejam sensíveis também através de indicadores demográficos tradicionalmente utilizados, como a esperança de vida ao nascer; os anos de vida perdidos; as taxas de mortalidade infantil; as taxas brutas de mortalidade e as taxas específicas de mortalidade.

No entanto, o exame da incidência de uma doença, por seu caráter multicausal, ou do padrão de morbimortalidade da população, por sua própria complexidade, se reportam de imediato a questões mais amplas.

No caso particular do aumento da incidência de neoplasias, por exemplo, é preciso considerar: 1) a qualidade dos alimentos, da água, do solo e do ar, incluindo a concentração de metais pesados, de aditivos, de corantes e de resíduos de produtos de limpeza, de combustíveis e de agrotóxicos; 2) a duração e intensidade da exposição aos mais variados compostos da indústria química contemporânea e 3) os aspectos comportamentais. Além disso, para uma rigorosa avaliação dos agravos das mudanças ambientais à saúde, devem-se levar em conta as alterações na incidência de óbitos por sexo, por idade e por grupos de causas, no contexto da transição epidemiológica (HOGAN, 2000a). 
$\mid 44$ |

O estudo da morbimortalidade no Brasil sob o enfoque...

A experiência de populações em estágios avançados da transição epidemiológica demonstra que na medida em que as doenças infecciosas e parasitárias recuam, as doenças degenerativas assumem maior importância, já que as pessoas sobrevivem até idades nas quais estão mais sujeitas a cânceres e a cardiopatias. Entretanto, há evidências de que essa transição acontece também porque paralelamente às conquistas da medicina, do saneamento básico e dos melhoramentos nutricionais, advindos do desenvolvimento territorial, social e econômico, houve profundas mudanças no comportamento ${ }^{3}$ humano e nas condições de exposição a substâncias originalmente não encontradas na natureza (HOGAN, 2007).

Esse artigo propõe uma discussão sobre alguns dos potenciais e dos limites da utilização de indicadores de morbimortalidade nos estudos demográficos e ambientais, salientando a necessidade de considerar, simultaneamente, questões relacionadas: 1) às transições demográfica e epidemiológica e 2) à transição urbanoindustrial; sinalizando possíveis caminhos para avanços no campo de estudos sobre P-A no Brasil.

\section{Transição demográfica, transição epidemiológica e indicadores de morbimortalidade no Brasil}

O aumento da esperança de vida ao nascer é um processo notável, hoje, em escala planetária. Sua ocorrência está associada tanto às mudanças nos níveis e padrões epidemiológicos da mortalidade quanto às alterações na estrutura etária da população.

Há uma clara associação entre a transição epidemiológica e a transição demográfica ${ }^{4}$, pois, em princípio, o declínio da

\footnotetext{
${ }^{3}$ Faz-se referência a mudanças: 1) na dieta, com a adição de açúcares, de gorduras, de alimentos refinados e de outros aditivos; e 2) no estilo de vida das pessoas, com maior incidência de sedentarismo, de tabagismo e de alcoolismo; por exemplo.

${ }^{4}$ A queda nas taxas de mortalidade, ao se combinar com uma posterior queda nas taxas de fecundidade, caracteriza o processo de transição demográfica, que
} 
mortalidade tende a concentrar-se seletivamente entre as doenças nutricionais, infecciosas e parasitárias, beneficiando, sobretudo, as crianças. Na medida em que diminui a mortalidade infantil e aumenta a esperança de vida, as doenças crônico-degenerativas e as causas violentas ganham em importância relativa entre as principais causas de morte, fenômeno que tende a se acentuar ao passo em que a estrutura populacional envelhece (CHAIMOWICZ, 1997).

A transição epidemiológica foi inicialmente descrita através de três estágios sucessivos: 1) a idade das pestes e da fome; 2) a idade do declínio das pandemias e 3) a idade das doenças degenerativas e por causas antrópicas. Na época em que esse modelo explicativo foi proposto, se acreditava que o terceiro estágio dessa transição equivaleria aos mais baixos níveis de mortalidade possíveis (OMRAN, 1971).

No entanto, dados internacionais passaram a indicar, em países desenvolvidos, posteriores quedas nos níveis de mortalidade. Partindo dessa constatação, Olshansky e Ault (1986), Rogers e Hackenberg (1987) e Omran (1998), dentre outros autores, reconheceram a necessidade de adicionar uma quarta etapa à teoria inicial da transição epidemiológica, referente a um período caracterizado: 1) pelo retardamento da mortalidade por cardiopatias; 2) pela emergência/reemergência de doenças ${ }^{5}$ infecciosas e parasitárias e 3) pela elevação da incidência de mortes violentas (homicídios, suicídios e acidentes de transporte).

Essa mudança, da terceira para a quarta fase da transição epidemiológica, tem sido atribuída a uma combinação de fatores, que vão desde os avanços na medicina, passando pela disseminação de infraestruturas e programas de saúde, pela

incide em alterações substanciais no ritmo de crescimento e na composição etária da população, conforme a clássica descrição de Notestein (1945).

${ }^{5}$ Referência ao surgimento de doenças como o HIV, as Hepatites B e C, o Ebola, a doença de Lyme, o Hantavírus e novas formas de E.Coli; e ao ressurgimento de doenças como o Cólera, a Malária, a Dengue, a Difteria, a Tuberculose e a doença de Chagas. 
|46|

O estudo da morbimortalidade no Brasil sob o enfoque...

redução dos fatores ambientais de agravos à saúde, para idosos, até a influência crescente de comportamentos individuais e de estilos de vida contemporâneos sobre as condições de morbimortalidade (LUSSIER; BOURBEAU; CHOINIÈRE; 2008).

Em países avançados, o retardamento da mortalidade por doenças degenerativas, principalmente por cardiopatias, indica que o aumento da mortalidade por tais doenças não é simplesmente uma consequência inevitável do envelhecimento populacional, já que se mostra relativamente prevenível, contexto no qual as mudanças ambientais, infraestruturais e comportamentais assumem importância significativa para a saúde pública (PRATA, 1992).

Seguindoessalinhaderaciocínio, operfildemorbimortalidade pode ser utilizado como um indicador relativamente sensível das condições de vida, do regime demográfico e do modelo de desenvolvimento sob os quais vive a população, sendo o resultado da interação entre diversos fatores (PRATA, 1992).

De fato, no Brasil, ocorreu um declínio significativo da taxa bruta de mortalidade, que passou de 15,3 (óbitos para cada mil habitantes) (1950-55) para 6,3 (2005-10). Tal mudança foi fortemente influenciada pelas quedas na taxa de mortalidade infantil, que passou de 134,8 (óbitos infantis para cada mil crianças nascidas vivas) (1950-55) para 23,6 (2005-10), incidindo na elevação da esperança de vida ao nascer, que passou de 51,0 (anos) (195055) para 72,4 (2005-10). Paralelamente, observa-se uma queda na taxa de fecundidade total, que passou de 6,2 (filhos por mulher em idade reprodutiva) (1950-55) para 1,9 (2005-2010), caracterizando a transição demográfica brasileira, conforme os dados apresentados na Tabela 1.

A transição demográfica incidiu em alterações significativas na estrutura etária da população brasileira, que passou de uma composição relativamente jovem (1980) para uma composição relativamente envelhecida (2010), conforme ilustrado pela Figura 1. 
TABELA 1 - Taxas Brutas de Mortalidade (TBM), Taxas de Mortalidade Infantil (TMI), Esperança de Vida ao Nascer (e0) e Taxas de Fecundidade Total (TFT) por períodos quinquenais, Brasil - 1950-2010

\begin{tabular}{c|c|c|c|c}
\hline Período & TBM & TMI & $\mathrm{e} 0$ & $\mathrm{TFT}$ \\
\hline $1950-1955$ & 15,3 & 134,8 & 51,0 & 6,2 \\
\hline $1955-1960$ & 13,9 & 122,0 & 53,5 & 6,2 \\
\hline $1960-1965$ & 12,5 & 109,5 & 55,9 & 6,2 \\
\hline $1965-1970$ & 11,0 & 100,2 & 57,9 & 5,4 \\
\hline $1970-1975$ & 9,9 & 90,6 & 59,8 & 4,7 \\
\hline $1975-1980$ & 9,0 & 78,9 & 61,8 & 4,3 \\
\hline $1980-1985$ & 8,3 & 63,3 & 63,5 & 3,8 \\
\hline $1985-1990$ & 7,4 & 52,4 & 65,5 & 3,1 \\
\hline $1990-1995$ & 6,8 & 42,5 & 67,5 & 2,6 \\
\hline $1995-2000$ & 6,5 & 34,1 & 69,4 & 2,5 \\
\hline $2000-2005$ & 6,4 & 27,4 & 71,0 & 2,3 \\
\hline $2005-2010$ & 6,3 & 23,6 & 72,4 & 1,9 \\
\hline
\end{tabular}

Fonte: United Nations, Department of Economic and Social Affairs, Population ivision (2013). World Population Prospects: The 2012 Revision, DVD Edition. 
$\mid 48$ |

O estudo da morbimortalidade no Brasil sob o enfoque...

FIGURA 1 - Distribuição etária da população por grupos quinquenais de idade (\%), Brasil - 1980/2010
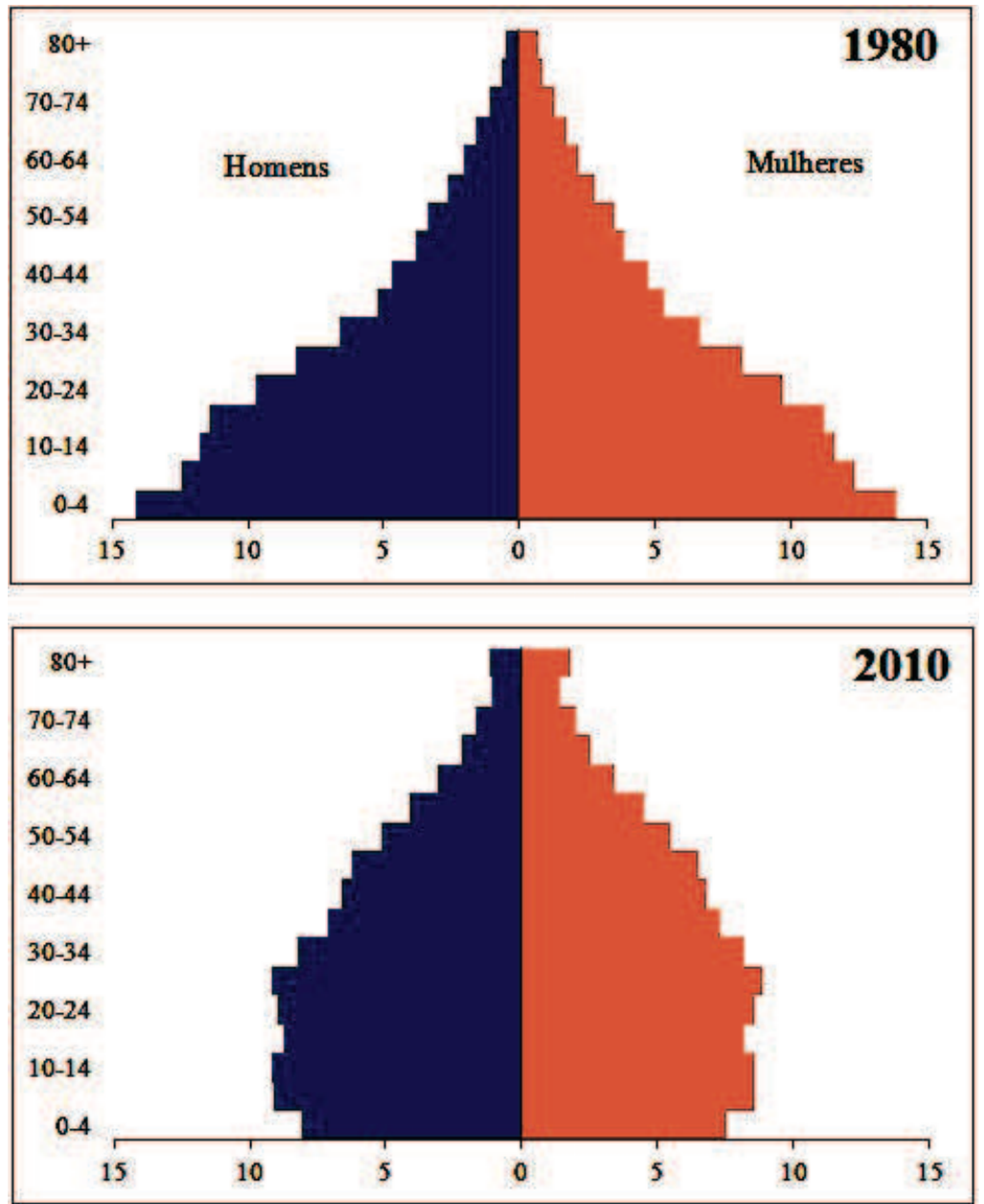

Fonte: IBGE, Censos Demográficos 1980 e 2010. 
Como já mencionado, no Brasil, as alterações na dinâmica demográfica e, por consequência, na estrutura etária da população, foram acompanhadas pela transição no perfil epidemiológico da mortalidade, com quedas na incidência de óbitos por doenças infecciosas e parasitárias, sobretudo, entre as crianças, e aumento da mortalidade por causas violentas e por doenças crônico-degenerativas, principalmente para jovens e idosos, respectivamente (SCHRAMM et. al, 2004; LEBRÃO, 2007).

Apesar desses resultados se aproximarem do esperado, no caso de uma população em processo de envelhecimento, é importante salientar que a transição epidemiológica brasileira não deve ser vista de maneira linear, já que traz como algumas de suas características: 1) a superposição de etapas, com aumento relativo da mortalidade por doenças não-transmissíveis e a persistência da morbimortalidade por doenças infecciosas e parasitárias; 2) uma contra-transição, relativa ao retorno de doenças transmissíveis, algumas das quais supostamente controladas e 3) a polarização epidemiológica, caracterizada pela persistência das desigualdades sociais e pela heterogeneidade de perfis epidemiológicos entre diferentes grupos populacionais (LEBRÃO, 2007).

Outro aspecto atípico e que chama a atenção para as especificidades da transição epidemiológica brasileira diz respeito aos elevadíssimos níveis de mortalidade por causas violentas, cujos diferenciais internos à população são evidentes, tanto por sexo e idade (MANETTA; ALVES, 2014) quanto por estrato social e local de residência (MINAYO, 2005 e 2009).

O estudo da elevação da mortalidade por causas violentas, durante as últimas décadas, demonstra fortes evidências de suas vinculações: 1) com o ambiente urbano; 2) com as más condições socioeconômicas e psicológicas da população jovem (CANIATO, 2008) e 3) com a precarização das condições de tráfego e de mobilidade nas grandes cidades e auto-estradas brasileiras (BACHIERI; BARROS, 2011); embora sua análise, sob a ótica das relações entre P-A, apareça ainda apenas como possibilidade. 
$|50|$

O estudo da morbimortalidade no Brasil sob o enfoque...

Urbanização, industrialização e agravos à saúde humana no Brasil

No início dos anos 1980, a sociedade brasileira, já significativamente urbanizada, se complexificou. Tanto seu sistema urbano como sua estrutura produtiva diversificaram-se, aguçando os desequilíbrios e as desigualdades sócio-espaciais, quando os ganhos em produtividade tiveram como causa e contrapartida o crescimento da oferta de bens e de serviços, às custas de um sensível agravo nas condições de vida para diferentes segmentos da população (FARIA, 1983).

Apesar dos benefícios registrados durante a transição urbano-industrial brasileira, um contexto diferenciado de agravos à saúde humana emergiu (AUGUSTO; CÂMARA, 2003), devido a uma série de processos, dentre os quais, se destacam: 1) a continuidade do crescimento e da concentração populacional em áreas urbanas, embora em ritmos arrefecidos, durante as últimas décadas (MARTINE; CAMARGO, 2013); 2) as mudanças na base econômico-produtiva nacional, tanto nas cidades quanto no campo (SANTOS; SILVEIRA, 2001); 3) a precarização do mercado de trabalho (ANTUNES; ALVES, 2004) e 4) a precarização das condições infraestruturais e ambientais intra-urbanas, para parcelas significativas da população.

A produção e a reprodução desse espaço urbano-industrial implicaram na emergência de um novo contexto de agravos à saúde da população, pois, apesar dos avanços sociais, econômicos e infraestruturais recentemente registrados (IBGE, 2002; 2012), problemas antigos ainda persistem, em inúmeras localidades urbanas brasileiras, como:1) a insuficiência da coleta e de tratamento de esgoto; 2) a existência de parcela significativa da coleta de lixo e de resíduos industriais sem destinação final apropriada; 3) deficiências na coleta, no tratamento e na distribuição de água e 5) lacunas nas redes de prestação de serviços sociais básicos.

No Brasil, embora tenham sido registradas melhorias na cobertura por serviços e infra-estururas de saneamento básico, desde a década de 1950 até o final do século passado, esse tipo 
de investimento ocorreu pontualmente, em localidades e períodos específicos. Em decorrência desse fato, a urbanização brasileira carrega ainda como marca um enorme déficit quanto ao acesso a esses serviços e infra-estruturas, principalmente no que se refere à coleta e tratamento de rejeitos domiciliares (Leoneti; Prado; Oliveira, 2011).

Hogan (1995) reconhece esses como alguns dos velhos problemas da urbanização brasileira, cuja demanda histórica por soluções representa uma dívida cumulativa entre o atraso na implantação desses serviços e infraestruturas e as necessidades sociais presentes.

Em países como o Brasil, caracterizados por uma urbanização precoce (MARTINE; McGRANAHAN, 2010) e incompleta (SANTOS, 1993), a população tende a conviver com situações de exclusão e de insatisfação das necessidades básicas diárias, cenário que produz uma exposição constante aos mais diversos agravos, devido, sobretudo, a uma estrutura desigual de oportunidades e ao acesso restrito aos melhores benefícios da urbanização e do crescimento econômico (KOWARICK, 1978; 2002; 2009).

Nessas condições, as dificuldades de inserção laboral, de qualificação e de obtenção de renda, são sobrepostas às más condições ambientais e domiciliares, configurando situações de elevada vulnerabilidade socioambiental (MARANDOLA JR.; HOGAN, 2006).

$\mathrm{O}$ resultado tem sido o aumento de pessoas sobrevivendo em condições insalubres, nos domicílios, espaços públicos e nos locais de trabalho (ROSSI-ESPAGNET; GOLDSTEIN; TABIBZEDE, 1991; GOUVEIA, 1999).

A questão do saneamento urbano foi uma das principais precursoras dessa discussão no Brasil (HELLER, 1998) e ainda se mantém relevante, particularmente no que diz respeito aos diferenciais inter-regionais e intra-urbanos da morbimortalidade infantil, especialmente por doenças transmissíveis (CARNEIRO et al., 2012; OLIVEIRA; SIMÕES, 2006).

A falta de saneamento básico tem sido reconhecida como componente capaz de propiciar o desencadeamento de epidemias, 
como as de dengue, por exemplo, conforme demonstra estudo de caso no município de Altamira (PA), onde a mobilidade populacional tende a estar relacionada com a inserção de novos sorotipos e com o início de novas epidemias, potencializadas por condições ambientais desfavoráveis (JOHANSEN; CARMO, 2012).

O mundo urbano-industrial tem passado por alterações significativas nas formas de organização do trabalho e na criação de novos produtos e procedimentos. Essas transformações implicaram na evolução dos agravos à saúde, tanto no ambiente de trabalho quanto no seu entorno, evidenciando uma complexificação crescente dos impactos dos processos produtivos e de ocupação territorial na saúde humana (PORTO, 2005; GUERRA et. al, 2005; FRANCO, 1993).

Em Cubatão, durante os anos 1980, o envenenamento por benzeno e a elevada incidência de doenças respiratórias e dermatológicas representaram evidências de casos extremos dos agravos da poluição ambiental à saúde. Entretanto, esses casos não esgotam as consequências negativas dessa exposição para a população humana (HOGAN, 1990; 1993), pois, como lembram Tambellini e Câmara (1998), com relação ao poluente, por sua elevada capacidade de modificação química, as possibilidades de alterações em seu poder patogênico devem ser ressaltadas.

As relações entre poluição atmosférica e a incidência de doenças respiratórias têm sido sistematicamente observadas no Brasil, notadamente para a população idosa, pois o aparelho respiratório, justamente pelas funções que desempenha, está particularmente exposto às alterações ambientais, tornando-se sede de agravos de intensidade variada (SOUZA; NETO, 2011; GIODA; GIODA, 2009; GOMES, 2002).

Apesar das dificuldades em distinguir, no registro da morbimortalidade por doenças respiratórias, os efeitos da poluição atmosférica, conclui-se que: 1) em geral, os efeitos nocivos da poluição, uma vez somados ao consumo do cigarro, têm ampliados seus potenciais de agravo à saúde e 2) os pobres tendem a sofrer de forma mais intensa os efeitos da poluição atmosférica, tanto no 
que diz respeito às condições insalubres de trabalho quanto às más condições ambientais domiciliares (HOGAN, 2000b).

Além dos aspectos gerais relacionados à falta de saneamento e à poluição ambiental, o processo de instalação das relações de produção capitalista no campo, particularmente observável no Centro-Oeste e na Amazônia, trouxeram mudanças significativas que determinaram o agravo do quadro endêmico e epidêmico de morbidades como a malária, em um processo que passa também pelo contágio da população em núcleos urbanos próximos às áreas de produção agrícola (BARBIERI, 2011).

O estabelecimento da malária em regiões de floresta tropical tem sido avaliado como resultado de processos interativos entre o homem e o ambiente, através do garimpo, da agropecuária e de atividades urbanas, que levam à ruptura do equilíbrio ecológico existente. Nesse caso, o fluxo de pessoas entre áreas de alta e baixa prevalência significa também a mobilidade da doença. Em áreas de colonização agrícola e pecuária, o estabelecimento de altos níveis de prevalência da malária tem sido facilitado pela abundância de vetores contaminados, pela precariedade das habitações e pela contaminação por exposição extradomiciliar, devido às atividades relacionadas à derrubada da floresta (BARBIERI, 2011).

De acordo com Monte-Mór (1986), é justamente durante os primeiros estágios de transformação do espaço natural em espaço construído que as condições ambientais se mostram mais propícias ao estabelecimento do ciclo da malária humana.

O conjunto dessas questões leva a crer que os impactos das mudanças ambientais adquirem importância crescente no padrão de morbimortalidade brasileiro, trazendo à tona considerações importantes ao campo de estudos sobre P-A, na medida em que um processo de transição urbano-industrial precoce, incompleto e excludente, avança no Brasil, tanto nas cidades quanto no campo, em um contexto também avançado da transição demográfica. 
154 |

O estudo da morbimortalidade no Brasil sob o enfoque...

\section{Considerações finais}

Do ponto de vista dos estudos sobre P-A, as especificidades da transição epidemiológica brasileira trazem considerações importantes sobre suas componentes socioeconômicas, comportamentais, demográficas e ambientais, estando alguns dos segmentos populacionais em ampla desvantagem com relação à sobreposição de agravos à saúde, principalmente no que se refere aos agravos que representam situações de relativo atraso social, sendo que algumas das potencialidades/dificuldades inerentes a essa análise devem ser ressaltadas.

As possibilidades mais imediatas de geração de dados demográficos e ambientais, específicos para localidades de interesse, principalmente através da realização de surveys, reafirmam a tendência em avaliar os impactos locais das mudanças ambientais, mesmo no caso de mudanças de caráter global, como as alterações climáticas, por exemplo, caminhando rumo a resultados mais precisos e evitando generalizações excessivas.

Entretanto, o reconhecimento das dificuldades na geração de dados demográficos e ambientais para um tratamento estatístico refinado, assim como as limitações das bases de dados disponíveis, salientam o potencial do padrão de morbimortalidade como indicador útil no campo de estudos sobre P-A, sobretudo, quando são reconhecidas melhorias na cobertura e na qualidade dos dados ${ }^{6}$ disponibilizados pelo Sistema de Informações sobre Mortalidade (SIM/DATASUS).

Feitas essas considerações, conclui-se sobre a utilidade atual dos indicadores de morbimortalidade no campo de estudos sobre P-A, desde que algumas precauções sejam tomadas, relativas: 1) ao reconhecimento do estágio contemporâneo do regime demográfico da população objeto de análise, incluindo sua composição etária e seus padrões predominantes de mobilidade espacial, no período

${ }^{6}$ Para uma discussão sobre a evolução na cobertura e na qualidade dos dados disponibilizados pelo SIM/DATASUS, ver: PAES (2005) e MELLO-JORGE, LAURENTI e GOTLIEB (2007). 
de referência doestudo; 2) ao reconhecimento dos principais eventos e processos de alteração ambiental, capazes de influenciarem significativamente as condições de sobrevivência da população; e 3) ao reconhecimento das escalas espaço-temporais mais adequadas e das bases de dados capazes de propiciar a produção de indicadores relevantes, em acordo com as especificidades de cada caso a ser avaliado.

\section{Referências}

ANTUNES, R.; ALVES, G. As mutações no mundo do trabalho na era da mundialização do capital. Educação e Sociedade. Campinas-SP, v. 25, n. 87, p. 335-351, 2004.

AUGUSTO, L. G. D. S.; CÂMARA, V. D. M. Saúde e ambiente: uma reflexão da Associação Brasileira de Pós-Graduação em Saúde Coletiva-ABRASCO. Revista Brasileira de Epidemiologia. São Paulo-SP, v. 6, n. 2, p. 87-94, 2003.

BACCHIERI, G.; BARROS, A. J. D. Acidentes de trânsito no Brasil de 1998 a 2010: muitas mudanças e poucos resultados. Revista de Saúde Pública. São Paulo-SP, v. 45, n. 5, p. 949-63, 2011.

BARBIERI, A. F. População, saúde e uso da terra: avaliação da prevalência de malária em uma região da Amazônia Brasileira. In: Seminário brasileiro de demografia da IUSSP, 1., 2011, Salvador. Anais... Salvador: IUSSP, 2011.

CANIATO, A. M. P. Violências e subjetividades. O indivíduo contemporâneo. Psicologia \& Sociedade. Belo Horizonte-MG, v. 20, n. 1, p. 16-32, 2008.

CARNEIRO, F. F. et al. Saúde ambiental e desigualdades: construindo indicadores para o desenvolvimento sustentável. Revista Ciência \& Saúde Coletiva. Rio de Janeiro-RJ, v. 17, n. 6, p. 1419-1425, 2012. 
CHAIMOWICZ, F. A saúde dos idosos brasileiros às vésperas do século XXI: problemas, projeções e alternativas. Revista de Saúde Pública. São Paulo, v. 31, n. 2, p. 184-200, 1997.

FARIA, V. E. Desenvolvimento, urbanização e mudanças na estrutura do emprego: a experiência brasileira dos últimos trinta anos. In. SORJ, B.; ALMEIDA, M. H. T. (Orgs.) Sociedade Política no Brasil Pós-64. São Paulo: Editora Brasiliense, 1983. p. 1-23.

FRANCO, T. Trabalho industrial e meio ambiente: a experiência do complexo industrial de Camaçari. In. MARTINE, G. (Org.). População, meio ambiente e desenvolvimento: verdades e contradições. Campinas: Editora da Unicamp, 1993. p. 60100.

GIODA, A.; GIODA, F. R. A influência da qualidade do ar nas doenças respiratórias. Revista Saúde e Ambiente. Joinville-SC, v. 7, n. 1, p. 15-23, 2009.

GOMES, M. J. M. Ambiente e pulmão. Jornal Brasileiro de Pneumologia. Brasília-DF, v. 28, n. 5, p. 261-269, 2002.

GOUVEIA, N. Saúde e meio ambiente nas cidades: os desafios da saúde ambiental. Saúde e sociedade. São Paulo-SP, v. 8, n. 1, p. 49-61, 1999.

GUERRA, M. R. et al. Risco de câncer no Brasil: tendências e estudos epidemiológicos mais recentes. Revista Brasileira de Cancerologia. Rio de Janeiro, v. 51, n. 3, p. 227-234, 2005.

HELLER, L. Relação entre saúde e saneamento na perspectiva do desenvolvimento. Ciência e Saúde Coletiva. Rio de Janeiro, v. 3, n. 2, p. 73-84, 1998.

HOGAN, D. J. A qualidade ambiental urbana: oportunidades para um novo salto. São Paulo em Perspectiva. São Paulo-SP, v. 9, n. 3, p. 17-23, 1995.

HOGAN, D. J. A relação entre população e ambiente: desafios para a demografia. In. TORRES, H. C., HELOISA (Orgs.). População 
e meio ambiente: debates e desafios. São Paulo: SENAC, 2000a. p. 21-52.

HOGAN, D. J. População e Meio Ambiente: a emergência de um novo campo de estudos. In. HOGAN, D. J. (Org.). Dinâmica populacional e mudança ambiental: cenários para o desenvolvimento brasileiro. Campinas: NEPO, 2007. p.13-58.

HOGAN, D. J. População, pobreza e poluição em Cubatão, São Paulo. In. MARTINE, G. (Org.). População, meio ambiente e desenvolvimento: verdades e contradições. Campinas: Ed. Unicamp, 1993. p. 101-103.

HOGAN, D. J. Quem paga o preço da poluição? Uma análise de residentes e migrantes pendulares em Cubatão. In: Encontro Nacional de Estudos Populacionais, 1990, Caxambu. Anais... Caxambú, MG: ABEP, 1990.

HOGAN, D. J. Redistribuição da população e meio ambiente: São Paulo e Centro-Oeste. Textos Nepo, n. 36. Núcleo de Estudos de População "Elza Berquó", Universidade Estadual de Campinas. Campinas, SP, $2000 \mathrm{~b}$.

IBGE. Censos Demográficos 1980 e 2010. Rio de Janeiro: IBGE, 1980 e 2010 .

IBGE. Pesquisa Nacional de Saneamento Básico. Rio de Janeiro: IBGE, 2002.

IBGE. Sintese de indicadores sociais: uma análise das condições de vida da população brasileira: 2012. Coleção Ibgeana: estudos e pesquisas. IBGE. Rio de Janeiro, RJ, 2012.

JOHANSEN, I. C.; CARMO, R. L. D. Dengue e falta de infraestrutura urbana na Amazônia brasileira: o caso de Altamira (PA). Novos Cadernos NAEA. Belém-PA, v. 15, n. 1, p. 179-208, 2012.

KOWARICK, L. A espoliação urbana. 1aㅡ ed. Rio de Janeiro: Editora Paz e Terra, 1978. 
KOWARICK, L. Viver em risco: sobre a vulnerabilidade no Brasil urbano. Novos Estudos Cebrap. São Paulo-SP, v. 63, p. 9-30, 2002.

KOWARICK, L. Viver em risco: sobre a vulnerabilidade socioeconômica e civil. 1ํㅡㄹ ed. São Paulo: Editora 34, 2009.

LEBRÃO, M. L. O envelhecimento no Brasil: aspectos da transição demográfica e epidemiológica. Cadernos Saúde Coletiva. Rio de Janeiro-RJ, v. 4, n. 17, p. 135-140, 2007.

LEONETI, A. B.; PRADO, E. L.; OLIVEIRA, S. V. W. B. Saneamento básico no Brasil: considerações sobre investimentos e sustentabilidade para o século XXI. Revista de Administração Pública. Rio de Janeiro, RJ, v. 45, n. 2, p. 331-348, 2011.

LUSSIER, M-H.; BOURBEAU, R.; CHOINIÈRE, R. Does the recent evolution of Canadian mortality agree with the epidemiologic transition theory? Demographic Research. Rostock-Alemanha, v. 18, p. 531-568, 2008.

LUTZ, W.; PRSKAWETZ, A.; SANDERSON, W. C. Introduction. Population and Development Review. Nova York-EUA, v. 28, p. 1-21, 2002.

MADEIRA, J. L. Malthus, Marx e o papel da população no desenvolvimento economico. In. IBGE. Lyra Madeira, um mestre da Demografia brasileira. Centro de Documentação e Disseminação de Informações. Rio de Janeiro, RJ: IBGE, 2008, p. 32-43.

MANETTA, A.; ALVES, J. E. D. Mortes violentas, inflexão na razão de sexo e impactos na esperança de vida da população: Argentina e Brasil (2001/2009). VI Congresso Latinoamericano de Estudos de População, 2014 Lima-Peru. Anais... Lima: ALAP, 2014. 
MARANDOLA JR, E.; HOGAN, D. J. As dimensões da vulnerabilidade. São Paulo em Perspectiva. São Paulo-SP, v. 20, n. 1, p. 33-43, 2006.

MARTINE, G.; CAMARGO, L. Crescimento e distribuição da população brasileira: tendências recentes. Revista Brasileira de Estudos de População. Belo Horizonte-MG, v. 1, n. 1/2, p. 99144, 2013.

MARTINE, G.; MCGRANAHAN, G. Brazil's Early Urban Transition: What Can It Teach Urbanizing Countries? $1^{\underline{a}}$ ed. Londres: IIED, 2010.

MELLO-JORGE, M.; LAURENTI, R.; GOTLIEB, S. L. D. Análise da qualidade das estatísticas vitais brasileiras: a experiência de implantação do SIM e do SINASC. Ciência E Saúde Coletiva. Rio de Janeiro, RJ, v. 12, n. 3, p. 643-54, 2007.

MINAYO, M. C. S. Violência: um problema para a saúde dos brasileiros. Impacto da violência na saúde dos brasileiros. Brasil. Brasília, DF: Ministério da Saúde: 2005, p. 9-33.

MINAYO, M. C. S. Seis características das mortes violentas no Brasil. Revista Brasileira de Estudos de População. Belo Horizonte-MG, v. 26, n. 1, p. 135-140, 2009.

MONTE-MOR, R. L. D. Malária e meio ambiente na Amazônia brasileira. I Seminário Latino-americano sobre População e Saúde, 1986, Campinas. Anais... Campinas,SP, 1986, p. 312328.

NOTESTEIN, F. W. Population: the long view. In: SCHULTZ, T. W. (Org.). Food for the World. Chicago: University of Chicago Press, 1945. p. 36-57.

OLIVEIRA, S.; SIMÕES, C. Meio ambiente urbano: mortalidade na infância, saneamento básico e políticas públicas. XV Encontro Nacional de Estudos Populacionais. Anais... Caxambu, MG: ABEP, 2006. 
$|60|$

O estudo da morbimortalidade no Brasil sob o enfoque...

OLSHANSKY, S. J.; AULT, A. B. The fourth stage of the epidemiologic transition: the age of delayed degenerative diseases. The Milbank Quarterly. San Diego-EUA, v. 64, n. 3, p. 355-391, 1986.

OMRAN, A. R. The epidemiologic transition theory revisited thirty years later. World Health Statistics Quarterly. San Diego-EUA, v. 51, n. 2-4, p. 99-119, 1998.

OMRAN, A. R. The epidemiologic transition: a theory of the epidemiology of population change. The Milbank Memorial Fund Quarterly. San Diego-EUA, v. 49, n. 4, p. 509-538, 1971.

UNITED NATIONS (UN). World Population Prospects: The 2012 Revision. Nova York: DESA, Population Division, 2012.

PAES, N. A. Avaliação da cobertura dos registros de óbitos dos estados brasileiros em 2000. Revista de Saúde Pública. São Paulo, SP, v. 39, n. 6, p. 882-90, 2005.

PORTO, M. F. Saúde do trabalhador e o desafio ambiental: contribuições do enfoque ecossocial, da ecologia política e do movimento pela justiça ambiental. Ciência \& Saúde Coletiva. Rio de Janeiro, RJ, v. 10, n. 4, p. 829-839, 2005.

PRATA, P. R. A transição epidemiológica no Brasil. Cadernos de Saúde Pública. Rio de Janeiro, v. 8, n. 2, p. 168-175, 1992.

ROGERS, R. G.; HACKENBERG, R. Extending epidemiologic transition theory: a new stage. Social biology, Utah-EUA, v. 34, n. 3-4, p. 234-243, 1987.

ROSSI-ESPAGNET, A.; GOLDSTEIN, G.; TABIBZEDEH, I. Urbanization and health in developing countries: a challenge for health for all. World health statistics quarterly. Nova YorkEUA, v. 44, n. 4, p. 186-247, 1991.

SANTOS, M. A urbanização brasileira. 1aㅡ ed. São Paulo, SP: Edusp, 1993. 
SANTOS, M.; SILVEIRA, M. L. O Brasil: território e sociedade no início do século XXI. $1^{\underline{a}}$ ed. Rio de Janeiro, RJ: Editora Record, 2001.

SCHRAMM, J. M. D. A. et al. Transição epidemiológica e o estudo de carga de doença no Brasil. Ciência \& Saúde Coletiva. Rio de Janeiro, RJ, v. 9, n. 4, p. 897-908, 2004.

SOUZA, C. G.; NETO, J. L. S. A. Ambiente e pulmão. Revista Hygeia. Uberlândia, MG, v. 7, n. 12, p. 31-45, 2011.

SWANSON, D.;SIEGEL, J.S. The methods and materials of demography. $1^{\underline{a}}$ ed. San Diego: Academic Press, 1976.

TAMBELLINI, A. T.; CÂMARA, V. D. M. A temática saúde e ambiente no processo de desenvolvimento do campo da saúde coletiva: aspectos históricos, conceituais e metodológicos. Ciência \& Saúde Coletiva. Rio de Janeiro, RJ, v. 3, n. 2, p. 47-59, 1998.

VANWEY, L. K.; OSTROM, E.; MERETSKY, V. Teorias subjacentes ao estudo de interações homem-ambiente. In: MORAN, E. F. e OSTROM, E. (Orgs.). Ecossistemas florestais: interação homemambiente. São Paulo, SP: Editora SENAC/EDUSP, 2009. 